


\section{COPY RIGHT}

$20204^{\text {th }}$ International Conference on Informatics and Computational Sciences (ICICoS) took place November 10-11, 2020

Copyright and Reprint Permission: Abstracting is permitted with credit to the source. Libraries are permitted to photocopy beyond the limit of U.S. copyright law for private use of patrons those articles in this volume that carry a code at the bottom of the first page, provided the per-copy fee indicated in the code is paid through Copyright Clearance Center, 222 Rosewood Drive, Danvers, MA 01923.

For other copying, reprint or republication permission, write to IEEE Copyrights Manager, IEEE Operations Center, 445 Hoes Lane, Piscataway, NJ 08854.

All rights reserved. Copyright (C) 2020 by IEEE.

ISBN: 978-1-7281-9526-1

IEEE catalog number: CFP20N15-ART 


\section{WELCOME SPEECH FROM THE GENERAL CHAIR OF ICICOS 2020}

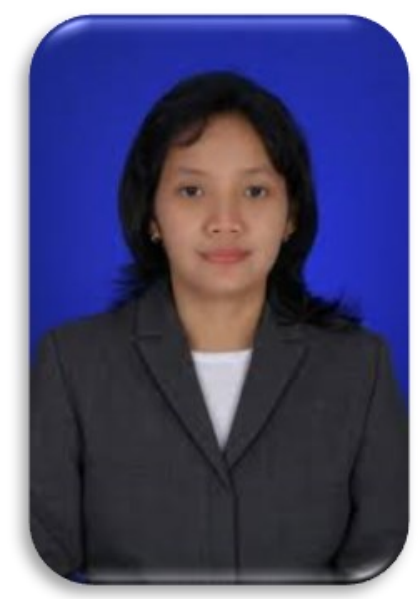

On behalf of the organizing committee, I am delighted to welcome all participants to the 4th International Conference on Informatics and Computational Sciences (ICICoS 2020). This conference is the fourth international conference held by Department of Informatics, Universitas Diponegoro in Semarang from November $10^{\text {th }}$ to November 11th, 2020.

In this conference, the committee decided to choose the following theme: "Accelerating Informatics and Computational Research for Society $5.0^{\prime \prime}$. This topic is in line with the concept of Society 5.0 which was introduced by Japan and inaugurated on January 21, 2019. Society 5.0 is a human-centered society that balances economic advancement with the resolution of social problems by a system that highly integrates cyberspace and physical space. This concept was born as a development of the 4.0 industrial revolution which is considered to degrade the role of humans. The aim of the conference is to provide an interactive international forum for sharing and exchanging information on the latest research in the area of computer sciences, informatics, computational science, and related field, which contribute to the society 5.0 .

Nearly 110 academicians, researcher, practitioner and presenters from 9 countries (Indonesia, United State of America, France, Austria, Australia, Japan, Timor Leste, Malaysia, and The Netherlands) have gathered in this event. In total, there are 84 active papers submitted to this conference. Each paper has been reviewed with tight criteria from our invited reviewers. Based on the review result, papers have been accepted, which lead to an acceptance rate of $47.6 \%$.

This conference will not be successful without extensive effort from many parties. First, I would like to thank all keynote speakers for allocating their valuable time to share their knowledge with us. I would also like to express my sincere gratitude to all participants who participate in this conference. Special acknowledgement should go to the Technical Program Committee Chairs, Members, and Reviewers for their thorough and timely reviewing of the papers. We would also like to thank our sponsors: IEEE Indonesia Section and Research and Society Service Institution Universitas Diponegoro, who have helped us to keep down the costs of ICICoS 2020 for all participants. Last but not least, recognition should also go to the Local Organizing Committee members who have put enormous effort and support for this conference.

At last, we hope that you have an enjoyable and inspiring moment during our conference. Thank you for your participation on ICICoS 2020.

Dr. Dinar Mutiara Kusumo Nugraheni, ST, M Info Tech(Comp)

General Chair of ICICoS 2020 


\section{PROGRAM SCHEDULE}

Tuesday, November $10^{\text {th }}, 2020$

\begin{tabular}{|c|c|c|c|c|}
\hline Time & Event & \multicolumn{3}{|c|}{ Event Details } \\
\hline $08.00-08.30$ & \multicolumn{4}{|c|}{ Participants entering virtual conference room } \\
\hline $08.30-08.35$ & \multirow{4}{*}{$\begin{array}{l}\text { Opening } \\
\text { Ceremony }\end{array}$} & \multicolumn{3}{|c|}{$\begin{array}{l}\text { Welcome Speech from the General Chair of ICICoS } 2020 \\
\text { (Dr. Dinar Mutiara KN, M. Info. Tech) }\end{array}$} \\
\hline $08.35-08.40$ & & \multicolumn{3}{|c|}{ Welcome Speech from IEEE IS } \\
\hline $08.40-08.50$ & & \multicolumn{3}{|c|}{$\begin{array}{l}\text { Welcome Speech from the Dean of Faculty of Science and } \\
\text { Mathematics, Universitas Diponegoro } \\
\text { (Prof. Dr. Widowati, M.Si) }\end{array}$} \\
\hline $08.50-09.00$ & & \multicolumn{3}{|c|}{$\begin{array}{l}\text { Opening Speech from the Rector Universitas Diponegoro } \\
\text { (Prof. Dr. Yos Johan Utama, S.H., M.Hum.) }\end{array}$} \\
\hline $09.00-10.30$ & Plenary I & \multicolumn{3}{|c|}{$\begin{array}{l}\text { - } \text { Keynote Speaker I: } \\
\text { Assoc Prof. AbdulRahman A. AlSewari } \\
\text { - Moderator: } \\
\text { Edy Suharto, ST, M.Kom }\end{array}$} \\
\hline $10.30-12.00$ & Plenary II & \multicolumn{3}{|c|}{ 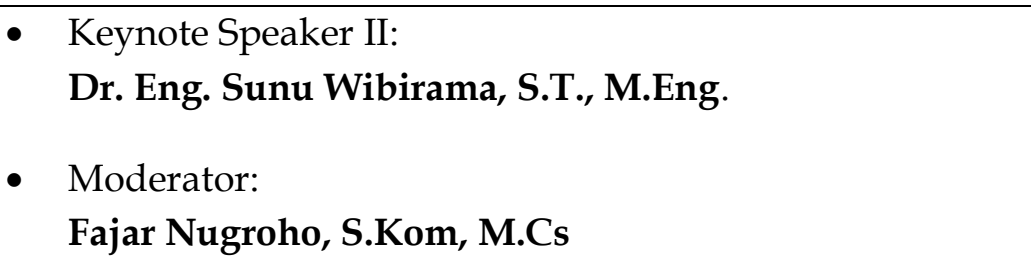 } \\
\hline $12.00-13.00$ & \multicolumn{4}{|c|}{ Break } \\
\hline & \multicolumn{4}{|c|}{ Parallel Session I } \\
\hline $13.00-14.30$ & $\begin{array}{l}\text { Room } 1 \\
\text { Information } \\
\text { System }\end{array}$ & $\begin{array}{l}\text { Room } 2 \\
\text { Software } \\
\text { Engineering, } \\
\text { Network and } \\
\text { Data Mining }\end{array}$ & $\begin{array}{c}\text { Room } 3 \\
\text { Machine Learning } \\
\text { and It's } \\
\text { Applications } 1\end{array}$ & $\begin{array}{c}\text { Room } 4 \\
\text { Machine Learning } \\
\text { and It's } \\
\text { Applications 2 }\end{array}$ \\
\hline $14.30-15.00$ & \multicolumn{4}{|c|}{ Break } \\
\hline
\end{tabular}




\begin{tabular}{|c|c|c|c|c|}
\hline \multirow[b]{2}{*}{$15.00-16.15$} & \multicolumn{4}{|c|}{ Parallel Session II } \\
\hline & $\begin{array}{c}\text { Room } 1 \\
\text { Information } \\
\text { System }\end{array}$ & $\begin{array}{l}\text { Room } 2 \\
\text { Software } \\
\text { Engineering, } \\
\text { Network and } \\
\text { Data Mining }\end{array}$ & $\begin{array}{c}\text { Room } 3 \\
\text { Machine Learning } \\
\text { and It's } \\
\text { Applications } 1\end{array}$ & $\begin{array}{c}\text { Room } 4 \\
\text { Machine Learning } \\
\text { and It's } \\
\text { Applications } 2\end{array}$ \\
\hline $16.15-16.30$ & \multicolumn{4}{|c|}{ Closing Ceremony } \\
\hline
\end{tabular}

Wednesday, November $11^{\text {th }}, 2020$

- Workshop 


\section{PROGRAM SCHEDULE - Parallel Session Schedule}

\begin{tabular}{|c|c|c|c|c|}
\hline \multicolumn{5}{|c|}{$\begin{array}{l}\text { Parallel Session - Room } 1 \\
\text { Information System }\end{array}$} \\
\hline Session & Time & Paper ID & Author & Title \\
\hline \multirow{6}{*}{ 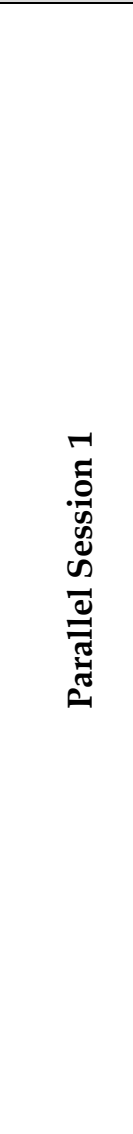 } & $\begin{array}{c}13.00 \\
- \\
13.15\end{array}$ & 1570657493 & $\begin{array}{l}\text { Meyliana Meyliana, Henry } \\
\text { Widjaja, Stephen Santoso, } \\
\text { Surjandy Surjandy, Erick } \\
\text { Fernando, Andreas Raharto } \\
\text { Condrobimo }\end{array}$ & $\begin{array}{l}\text { Improving the Quality of Learning } \\
\text { Management System (LMS) based } \\
\text { on Student Perspectives Using } \\
\text { UTAUT2 and Trust Model }\end{array}$ \\
\hline & $\begin{array}{c}13.15 \\
- \\
13.30\end{array}$ & 1570660660 & $\begin{array}{l}\text { Aulia-Absari Khalil, Meyliana, } \\
\text { Achmad Nizar Hidayanto, } \\
\text { Harjanto Prabowo }\end{array}$ & $\begin{array}{l}\text { Identification of Factor Affecting } \\
\text { Continuance Usage Intention of } \\
\text { mHealth Application }\end{array}$ \\
\hline & $\begin{array}{c}13.30 \\
- \\
13.45\end{array}$ & 1570669017 & $\begin{array}{l}\text { Julius Galih Prima Negara, } \\
\text { Djoko Budiyanto Setyohadi, } \\
\text { Pranowo }\end{array}$ & $\begin{array}{l}\text { Uncertainty Avoidance and } \\
\text { Individualism Collectivism on } \\
\text { Acceptance of Smart City Mobile } \\
\text { Applications }\end{array}$ \\
\hline & $\begin{array}{c}13.45 \\
- \\
14.00\end{array}$ & 1570670167 & $\begin{array}{l}\text { Zerlita Fahdha Pusdiktasari, } \\
\text { Rahma Fitriani, Eni } \\
\text { Sumarminingsih }\end{array}$ & $\begin{array}{l}\text { The Outlierness Degree of Spatial } \\
\text { Observations in the Presence of } \\
\text { Spatial Outliers, Simulation Study } \\
\text { using Average Difference Algorithm }\end{array}$ \\
\hline & $\begin{array}{c}14.00 \\
- \\
14.15\end{array}$ & 1570670570 & $\begin{array}{l}\text { Ragil Saputra, Tusta Rayana, } \\
\text { Satriyo Adhy, Nurdin Bahtiar, } \\
\text { Melnyi Timu }\end{array}$ & $\begin{array}{l}\text { Success Factor Affecting M- } \\
\text { Learning Implementation: } \\
\text { Perspective of Students }\end{array}$ \\
\hline & $\begin{array}{c}14.15 \\
- \\
14.30\end{array}$ & 1570671361 & $\begin{array}{l}\text { Aang Kisnu Darmawan, Daniel } \\
\text { Siahaan, Tony Dwi Susanto, } \\
\text { Hoiriyah Hoiriyah, Busro } \\
\text { Umam Achmad Nizar } \\
\text { Hidayanto, A'ang Subiyakto, } \\
\text { Miftahul Walid, Iwan Santosa }\end{array}$ & $\begin{array}{l}\text { Hien's Framework for Examining } \\
\text { Information System Quality of } \\
\text { Mobile-based Smart Regency } \\
\text { Service in Madura Island Districts }\end{array}$ \\
\hline & $\begin{array}{c}14.30 \\
- \\
15.00\end{array}$ & \multicolumn{3}{|c|}{ COFFEE BREAK } \\
\hline \multirow{3}{*}{ 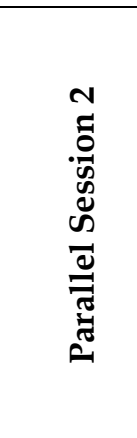 } & $\begin{array}{c}15.00 \\
- \\
15.15\end{array}$ & 1570674375 & $\begin{array}{l}\text { Dinar Mutiara Kusumo } \\
\text { Nugraheni, Amabel } \\
\text { Hadisoewono, Beta Noranita }\end{array}$ & $\begin{array}{l}\text { Continuance Intention to Use (CIU) } \\
\text { on Technology Acceptance Model } \\
\text { (TAM) for m-payment (case study: } \\
\text { TIX ID) }\end{array}$ \\
\hline & $\begin{array}{c}15.15 \\
- \\
15.30 \\
\end{array}$ & 1570677311 & $\begin{array}{l}\text { Ragil Saputra, Satriyo Adhy, } \\
\text { Nurdin Bahtiar, Nauli Isnaini, } \\
\text { Zaenal Abidin, Edy Suharto }\end{array}$ & $\begin{array}{l}\text { Factors Influencing Student's } \\
\text { Adoption of E-Learning in } \\
\text { Indonesian Secondary Schools }\end{array}$ \\
\hline & $\begin{array}{c}15.30 \\
- \\
15.45\end{array}$ & 1570677382 & $\begin{array}{l}\text { Indra Waspada, Riyanarto } \\
\text { Sarno }\end{array}$ & $\begin{array}{l}\text { An Improved Method of Graph Edit } \\
\text { Distance for Business Process Model } \\
\text { Similarity Measurement }\end{array}$ \\
\hline
\end{tabular}




\begin{tabular}{|c|c|c|c|c|}
\hline & & & $\begin{array}{r}\text { Parallel Session - Room } 2 \\
\text { Engineering, Network and D }\end{array}$ & Mining \\
\hline Session & Time & Paper ID & Author & Title \\
\hline \multirow{6}{*}{ 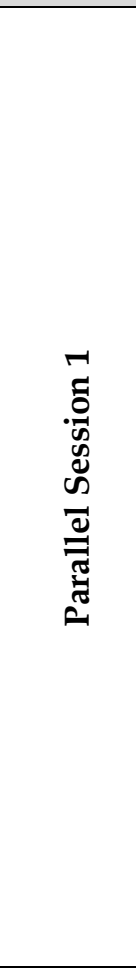 } & $\begin{array}{c}13.00 \\
- \\
13.15\end{array}$ & 1570655432 & $\begin{array}{l}\text { Zharfan Akbar Andriawan, } \\
\text { Satriawan Rasyid Purnama, } \\
\text { Ricko Ricko, Adam Sukma } \\
\text { Darmawan, Adi Wibowo, Aris } \\
\text { Sugiharto, Feri Wijayanto }\end{array}$ & $\begin{array}{l}\text { Prediction of Hotel Booking } \\
\text { Cancellation using CRISP-DM }\end{array}$ \\
\hline & $\begin{array}{c}13.15 \\
- \\
13.30\end{array}$ & 1570666102 & $\begin{array}{l}\text { Dhiyaussalam, Adi Wibowo, } \\
\text { Fajar Agung Nugroho, Eko Adi } \\
\text { Sarwoko, I Made Agus Setiawan }\end{array}$ & $\begin{array}{l}\text { Classification of Headache Disorder } \\
\text { Using Random Forest Algorithm }\end{array}$ \\
\hline & $\begin{array}{c}13.30 \\
- \\
13.45\end{array}$ & 1570669271 & $\begin{array}{l}\text { Suryo Santoso, Satriyo Adhy, } \\
\text { Nurdin Bahtiar, Indra Waspada }\end{array}$ & $\begin{array}{l}\text { Development of The Smart Chicken } \\
\text { Eggs Incubator Based on Internet of } \\
\text { Things Using The Object Oriented } \\
\text { Analysis and Design Method }\end{array}$ \\
\hline & $\begin{array}{c}13.45 \\
- \\
14.00\end{array}$ & 1570669787 & $\begin{array}{l}\text { Khadijah, Amazona Adorada, } \\
\text { Panji Wisnu Wirawan, Kabul } \\
\text { Kurniawan }\end{array}$ & $\begin{array}{l}\text { The Comparison of Feature } \\
\text { Selection Methods in Software } \\
\text { Defect Prediction }\end{array}$ \\
\hline & $\begin{array}{c}14.00 \\
- \\
14.15\end{array}$ & 1570670150 & $\begin{array}{l}\text { Zharfan Akbar Andriawan, } \\
\text { Ramadhan Pratama, Khadijah }\end{array}$ & $\begin{array}{l}\text { Usability Testing of Multifinance } \\
\text { Mobile Application for End- } \\
\text { Customer Case Study: PT. XYZ }\end{array}$ \\
\hline & $\begin{array}{c}14.15 \\
- \\
14.30\end{array}$ & 1570670259 & $\begin{array}{l}\text { Ahmad Saikhu, Agung Teguh } \\
\text { Setyadi, Yudhi Purwananto, } \\
\text { Arya Yudhi Wijaya }\end{array}$ & $\begin{array}{l}\text { Spatio-Temporal Recurrent Neural } \\
\text { Networks Modeling for Number of } \\
\text { Users Prediction on Wireless Traffic } \\
\text { Networks }\end{array}$ \\
\hline & $\begin{array}{c}14.30 \\
- \\
15.00\end{array}$ & \multicolumn{3}{|c|}{ COFFEE BREAK } \\
\hline \multirow{4}{*}{ 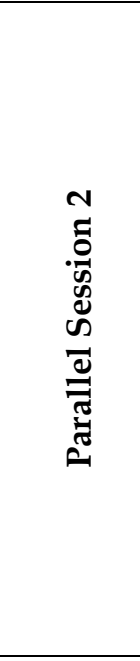 } & $\begin{array}{c}15.00 \\
- \\
15.15\end{array}$ & 1570673406 & $\begin{array}{l}\text { Busro Umam, Aang Kisnu } \\
\text { Darmawan, Anwari Anwari, } \\
\text { Iwan Santosa, Miftahul Walid, } \\
\text { Achmad Nizar Hidayanto }\end{array}$ & $\begin{array}{l}\text { Mobile-based Smart Regency } \\
\text { Adoption with TOE framework: An } \\
\text { empirical inquiry from Madura } \\
\text { Island Districts }\end{array}$ \\
\hline & $\begin{array}{l}15.15 \\
- \\
15.30\end{array}$ & 1570677016 & $\begin{array}{l}\text { Amarudin Amarudin, Ridi } \\
\text { Ferdiana, Widy Widyawan }\end{array}$ & $\begin{array}{l}\text { A Systematic Literature Review of } \\
\text { Intrusion Detection System for } \\
\text { Network Security: Research Trends, } \\
\text { Datasets and Methods }\end{array}$ \\
\hline & $\begin{array}{c}15.30 \\
- \\
15.45\end{array}$ & 1570677148 & $\begin{array}{l}\text { Muhamad Aldy Bintang, Ruki } \\
\text { Harwahyu, Riri Fitri Sari }\end{array}$ & $\begin{array}{l}\text { SMARIoT: Augmented Reality for } \\
\text { Monitoring System of Internet of } \\
\text { Things using EasyAR }\end{array}$ \\
\hline & $\begin{array}{l}15.45 \\
- \\
16.00\end{array}$ & 1570677427 & Flora Poecze, A Min Tjoa & $\begin{array}{l}\text { Meta-analytical considerations for } \\
\text { gamification in higher education: } \\
\text { existing approaches and future } \\
\text { research agenda }\end{array}$ \\
\hline
\end{tabular}




\begin{tabular}{|c|c|c|c|c|}
\hline & & & $\begin{array}{c}\text { Parallel Session - Room } 3 \\
\text { ine Learning and Its Applica }\end{array}$ & \\
\hline Session & Time & Paper ID & Author & Title \\
\hline \multirow{6}{*}{ 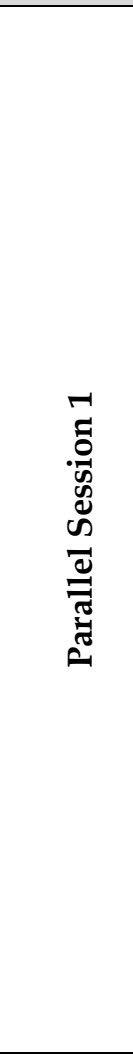 } & $\begin{array}{c}13.00 \\
- \\
13.15\end{array}$ & 1570660735 & $\begin{array}{l}\text { Devvi Sarwinda, Alhadi } \\
\text { Bustaman, Radifa H. Paradisa, } \\
\text { Terry Argyadiva, Wibowo } \\
\text { Mangunwardoyo }\end{array}$ & $\begin{array}{l}\text { Analysis of Deep Feature } \\
\text { Extraction for Colorectal Cancer } \\
\text { Detection }\end{array}$ \\
\hline & $\begin{array}{c}13.15 \\
- \\
13.30 \\
\end{array}$ & 1570670088 & $\begin{array}{l}\text { Agus Subhan Akbar, Chastine } \\
\text { Fatichah, Nanik Suciati }\end{array}$ & $\begin{array}{l}\text { Simple MyUnet3D for BraTS } \\
\text { Segmentation }\end{array}$ \\
\hline & $\begin{array}{c}13.30 \\
- \\
13.45\end{array}$ & 1570673146 & $\begin{array}{l}\text { Suherwin, Zahir Zainuddin, } \\
\text { Amil Ahmad Ilham }\end{array}$ & $\begin{array}{l}\text { The Performance of Face } \\
\text { Recognition Using the } \\
\text { Combination of Viola-Jones, Local } \\
\text { Binary Pattern Histogram and } \\
\text { Euclidean Distance }\end{array}$ \\
\hline & $\begin{array}{c}13.45 \\
- \\
14.00\end{array}$ & 1570674176 & $\begin{array}{l}\text { Eko Prasetyo, Nanik Suciati, } \\
\text { Chastine Fatichah }\end{array}$ & $\begin{array}{l}\text { A Comparison of YOLO and Mask } \\
\text { R-CNN for Segmenting Head and } \\
\text { Tail of Fish }\end{array}$ \\
\hline & $\begin{array}{c}14.00 \\
- \\
14.15\end{array}$ & 1570674320 & $\begin{array}{l}\text { Puteri Khatya Fahira Ari, Zulia } \\
\text { Putri Rahmadhani, Petrus } \\
\text { Mursanto, Ari Wibisono, Hanif } \\
\text { Arief Wisesa }\end{array}$ & $\begin{array}{l}\text { Classical Machine Learning } \\
\text { Classification for Javanese } \\
\text { Traditional Food Image }\end{array}$ \\
\hline & $\begin{array}{c}14.15 \\
- \\
14.30\end{array}$ & 1570676401 & $\begin{array}{l}\text { Daffa Muammar, Priyo Sidik } \\
\text { Sasongko, Sukmawati Nur } \\
\text { Endah, Retno Kusumaningrum, } \\
\text { Rismiyati, Khadijah, Lestari } \\
\text { Handayani }\end{array}$ & $\begin{array}{l}\text { Garbage Image Segmentation } \\
\text { Using Combination of } \\
\text { Thresholding Algorithms and } \\
\text { Pyramid Scene Parsing Network }\end{array}$ \\
\hline \multirow{6}{*}{ 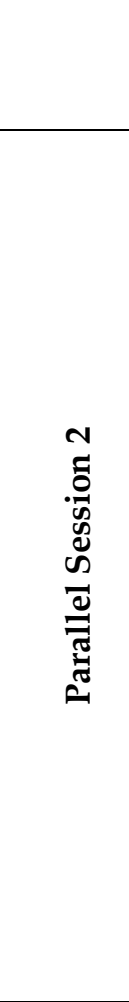 } & $\begin{array}{c}14.30 \\
- \\
15.00\end{array}$ & \multicolumn{3}{|c|}{ COFFEE BREAK } \\
\hline & $\begin{array}{c}15.00 \\
- \\
15.15\end{array}$ & 1570676489 & $\begin{array}{l}\text { I Wayan Agus Surya Darma, } \\
\text { Nanik Suciati, Daniel Siahaan }\end{array}$ & $\begin{array}{l}\text { Balinese Carving Recognition using } \\
\text { Pre-Trained Convolutional Neural } \\
\text { Network }\end{array}$ \\
\hline & $\begin{array}{c}15.15 \\
- \\
15.30\end{array}$ & 1570676888 & $\begin{array}{l}\text { Ni Putu Sutramiani, Nanik } \\
\text { Suciati, Daniel Siahaan }\end{array}$ & $\begin{array}{l}\text { Transfer Learning on Balinese } \\
\text { Character Recognition of Lontar } \\
\text { Manuscript Using MobileNet }\end{array}$ \\
\hline & $\begin{array}{c}15.30 \\
- \\
15.45\end{array}$ & 1570677274 & $\begin{array}{l}\text { Urratul Aqyuni, Rismiyati, } \\
\text { Sukmawati Nur Endah, Priyo } \\
\text { Sidik Sasongko, Retno } \\
\text { Kusumaningrum, Khadijah, } \\
\text { Hanif Rasyidi }\end{array}$ & $\begin{array}{l}\text { Waste Image Segmentation Using } \\
\text { Convolutional Neural Network } \\
\text { Encoder-Decoder with SegNet } \\
\text { Architecture }\end{array}$ \\
\hline & $\begin{array}{c}15.45 \\
- \\
16.00\end{array}$ & 1570677290 & $\begin{array}{l}\text { Tiani Tiara Putri, Ema } \\
\text { Rachmawati, Febryanti Sthevanie }\end{array}$ & $\begin{array}{l}\text { Indonesian Ethnicity Recognition } \\
\text { Based on Face Image Using } \\
\text { Uniform Local Binary Pattern } \\
\text { (ULBP) and Color Histogram }\end{array}$ \\
\hline & $\begin{array}{c}16.00 \\
- \\
16.15\end{array}$ & 1570677356 & $\begin{array}{l}\text { Adita Putri Puspaningrum, } \\
\text { Retno Kusumaningrum, } \\
\text { Sukmawati Nur Endah, } \\
\text { Khadijah, Rismiyati, Priyo Sidik } \\
\text { Sasongko, Ferda Ernawan }\end{array}$ & $\begin{array}{l}\text { Waste Classification Using Support } \\
\text { Vector Machine with SIFT-PCA } \\
\text { Feature Extraction }\end{array}$ \\
\hline
\end{tabular}


Machine Learning and Its Applications 2

\begin{tabular}{|c|c|c|c|c|}
\hline Session & Time & Paper ID & Author & Title \\
\hline \multirow{7}{*}{ 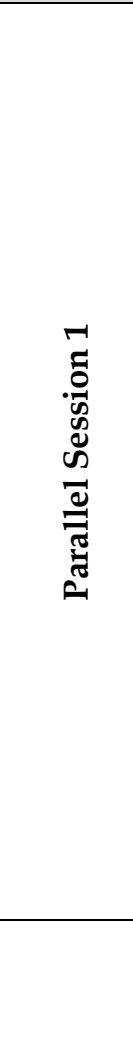 } & $\begin{array}{c}13.00 \\
- \\
13.15\end{array}$ & 1570666720 & $\begin{array}{l}\text { Cuk Tho, Harco Leslie Hendric } \\
\text { Spits Warnars, Benfano Soewito, } \\
\text { Ford Lumban Gaol }\end{array}$ & $\begin{array}{l}\text { Code-mixed Sentiment Analysis } \\
\text { using Machine Learning Approach - } \\
\text { A Systematic Literature Review }\end{array}$ \\
\hline & $\begin{array}{c}13.15 \\
- \\
13.30\end{array}$ & 1570670508 & $\begin{array}{l}\text { Muhammad Rizky Ramadhan, } \\
\text { Sukmawati Nur Endah, } \\
\text { Aprinaldi Bin Jasa Mantau }\end{array}$ & $\begin{array}{l}\text { Implementation of Textrank } \\
\text { Algorithm in Product Review } \\
\text { Summarization }\end{array}$ \\
\hline & $\begin{array}{c}13.30 \\
- \\
13.45\end{array}$ & 1570674565 & $\begin{array}{l}\text { Moh Abdul Latief, Alhadi } \\
\text { Bustamam, Titin Siswantining }\end{array}$ & $\begin{array}{l}\text { Performance Evaluation XGBoost in } \\
\text { Handling Missing Value on } \\
\text { Classification of Hepatocellular } \\
\text { Carcinoma Gene Expression Data }\end{array}$ \\
\hline & $\begin{array}{c}13.45 \\
- \\
14.00\end{array}$ & 1570674664 & $\begin{array}{l}\text { Ika Marta Sari, Saskya } \\
\text { Soemartojo, Titin Siswantining, } \\
\text { Devvi Sarwinda }\end{array}$ & $\begin{array}{l}\text { Mining Biological Information from } \\
\text { 3D Medulloblastoma Cancerous } \\
\text { Gene Expression Data Using } \\
\text { TimesVector Triclustering Method }\end{array}$ \\
\hline & $\begin{array}{c}14.00 \\
- \\
14.15\end{array}$ & 1570674674 & $\begin{array}{l}\text { Dea Siska, Titin Siswantining, } \\
\text { Devvi Sarwinda, Saskya } \\
\text { Soemartojo }\end{array}$ & $\begin{array}{l}\text { Triclustering Algorithm for 3D Gene } \\
\text { Expression Data Analysis using } \\
\text { Order Preserving Triclustering } \\
\text { (OPTricluster) }\end{array}$ \\
\hline & $\begin{array}{c}14.15 \\
- \\
14.30\end{array}$ & 1570676322 & $\begin{array}{l}\text { Agung Wiratmo, Chastine } \\
\text { Fatichah }\end{array}$ & $\begin{array}{l}\text { Assessment of Indonesian Short } \\
\text { Essay using Transfer Learning } \\
\text { Siamese Dependency Tree-LSTM }\end{array}$ \\
\hline & $\begin{array}{c}14.30 \\
- \\
15.00\end{array}$ & \multicolumn{3}{|c|}{ COFFEE BREAK } \\
\hline \multirow{4}{*}{ 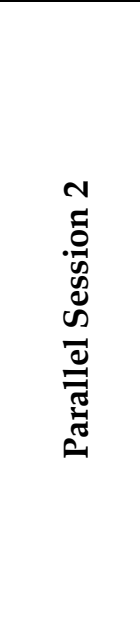 } & $\begin{array}{c}15.00 \\
- \\
15.15\end{array}$ & 1570676889 & $\begin{array}{l}\text { Sayyidah Hanifah Putri, Adhi } \\
\text { Harmoko Saputro }\end{array}$ & $\begin{array}{l}\text { Design of Convolutional Neural } \\
\text { Network Modeling for Low-Density } \\
\text { Lipoprotein (LDL) Levels } \\
\text { Measurement Based on Iridology }\end{array}$ \\
\hline & $\begin{array}{c}15.15 \\
- \\
15.30\end{array}$ & 1570676890 & $\begin{array}{l}\text { Dyla Velia, Adhi Harmoko } \\
\text { Saputro }\end{array}$ & $\begin{array}{l}\text { Designing Diabetes Mellitus } \\
\text { Detection System Based on } \\
\text { Iridology with Convolutional } \\
\text { Neural Network Modeling }\end{array}$ \\
\hline & $\begin{array}{c}15.30 \\
- \\
15.45\end{array}$ & 1570677039 & $\begin{array}{l}\text { Rismiyati, Sukmawati Nur } \\
\text { Endah, Khadijah, Ilman Nabil } \\
\text { Shiddiq }\end{array}$ & $\begin{array}{l}\text { Xception Architecture Transfer } \\
\text { Learning for Garbage Classification }\end{array}$ \\
\hline & $\begin{array}{c}15.45 \\
- \\
16.00\end{array}$ & 1570677362 & $\begin{array}{l}\text { Sutikno Sutikno, Khairul Anam, } \\
\text { Azmi Saleh }\end{array}$ & $\begin{array}{l}\text { Voice controlled wheelchair for } \\
\text { disabled patients based on CNN \& } \\
\text { LSTM }\end{array}$ \\
\hline
\end{tabular}




\section{COMMITTEES}

\section{Steering Committee:}

- Prof. Widowati, Universitas Diponegoro, ID

- Prof. W. Jatmiko, Universitas Indonesia, ID

- $\quad$ Prof. J. Jamari, Universitas Diponegoro, ID

- R. Kusumaningrum, Universitas Diponegoro, ID

- Kurnianingsih, Politeknik Negeri Semarang, ID

- NA. Setiawan, Universitas

\section{General Chair:}

- $\quad$ DMK. Nugraheni, Universitas Diponegoro, ID

\section{General Co-chairs:}

- PW. Wirawan, Universitas Diponegoro, ID

- $\quad$ R. Saputra, Universitas Diponegoro, ID

\section{Secretary:}

- $\quad$ S. Adhy, Universitas Diponegoro, ID

- SN. Endah, Universitas Diponegoro, ID

\section{Finance:}

- B. Noranita, Universitas Diponegoro, ID

- Khadijah, Universitas Diponegoro, ID

\section{Program Chair:}

- FA. Nugroho, Universitas Diponegoro, ID

\section{Publication Chair:}

- Rismiyati, Universitas Diponegoro, ID

- $\quad$ E. Suharto, Universitas Diponegoro, ID

\section{Technical Program Committee Chairs:}

- A. Wibowo, Universitas Diponegoro, ID

- MA. Riyadi, Universitas Diponegoro, ID

\section{Technical Program Committee Member:}

- $\quad$ AH. Ali, Universiti Kuala Lumpur, MY

- A. Alsobeh, Yarmouk University, JOR

- A. Antonyova, University of Prešov in Prešov, SVK

- A. Azizan, Universiti Teknologi Malaysia (UTM), MY

- A. Bagwari, IEEE Member, UTU, IN

- A. Ghasempour, ICT Faculty, USA

- A. Hamad, University of Baghdad, IRQ 
- A. Miry, Al-Mustansiriyah University, IRQ

- A. Mobashsher, The University of Queensland, AU

- $\quad$ AM. Nasrabadi, Shahed University, IRN

- A. Mukherjee, Jiangsu University, CN

- A. Najmurrokhman, Universitas Jenderal Achmad Yani, ID

- AP. Widodo, Diponegoro University, ID

- A. Rafiei, University of Technology Sydney, AU

- A. Sallam, Taiz University, YEM

- A. Sharma, Quantum University, Roorkee, Uttarakhand, IND

- AS. Shibghatullah, UCSI University, MY

- A. Sunyoto, Universitas Amikom Yogyakarta, ID

- A. Testa Ministry of Economy and Finance, ITA

- A. Wibowo, Diponegoro University, ID

- B. Jiang, The City University of New York, USA

- B. Warsito, Diponegoro University, ID

- C. Kerdvibulvech, National Institute of Development Administration, THA

- C. Sonagiri, Institute of Aeronautical Engineering, IND

- C. Yuen, Singapore University of Technology and Design, SG

- D. Andriana, Indonesian Institute of Sciences, ID

- D. Chisanga, The University of Zambia, ZMB

- D. Ciuonzo, University of Naples Federico II, ITA

- DH. Fudholi, Universitas Islam Indonesia, ID

- D. Krstić, Faculty of Electronic Engineering, University of Niš, SRB

- $\quad$ DMK. Nugraheni, Universitas Diponegoro, ID

- D. Nurjanah, Telkom University, ID

- D. Wijaya, Telkom University, ID

- $\quad$ E. Babulak, Liberty University, USA

- E. Jiménez Macías, University of La Rioja, ESP

- $\quad$ E. Kaburuan, BINUS University, ID

- $\quad$ E. Werbin, Universidad Nacional de Cordoba, ARG

- F. Aadil, COMSATS University Islamabad Attock Campus, PAK

- $\quad$ F. Farikin, Diponegoro University, ID

- G. Abbas, GIK Institute of Engineering Sciences \& Technology, PAK

- G. Dekoulis, Aerospace Engineering Institute (AEI), CYP

- G. Palmerini, Sapienza Università di Roma, ITA

- G. Tambouratzis, Institute for Language \& Speech Processing, GRC

- H. Abdulkareem, Al-Nahrain University, IRQ

- H. Alasadi, IRAQ- BASRA, IRQ

- H. El Abed, Technical Trainers College (TTC), SA

- H. Khattak, COMSATS University, Islamabad, PAK

- $\mathrm{H} . \mathrm{Ng}$, Multimedia University, MY

- H. Pan, New Jersey Institute of Technology, USA

- H. Pardede, Indonesian Institute of Sciences, ID

- HLH. Spits Warnars, Bina Nusantara University, ID

- H. Toba, Maranatha Christian University, ID 
- I. Song, Korea Advanced Institute of Science and Technology, KOR

- I. Yulita, Universitas Padjadjaran, ID

- J. Ali, University of Technology, IRQ

- J. Chin, Multimedia University, MY

- J. Suba, University of the Assumption, PHL

- K. Al-Saedi, Mustansiriyah University, IRQ

- K. Hopkinson, Air Force Institute of Technology, USA

- K. Kabassi, Ionian University, GRC

- K. Majumder, West Bengal University of Technology, IND

- L. Boubchir, University of Paris 8, FR

- MA. Ahmad, Universiti Malaysia Pahang, MY

- MKI. Ahmad, Universiti Tun Hussein Onn Malaysia, my

- M. Chen, Electrical Engineering, Da-Yeh University, TW

- M. Hasibuan, University Gadjah Mada, ID

- M. Koyimatu, Universitas Pertamina, ID

- M. Mustaffa, Universiti Putra Malaysia, MY

- M. Nyirenda, University of Zambia, ZM

- M. Riasetiawan, Universitas Gadjah Mada, ID

- M. Riyadi, Diponegoro University, ID

- M. Tuloli, Universitas Negeri Gorontalo, ID

- M. Younis, University of Baghdad, IRQ

- M. Yusuf, University of Trunojoyo, ID

- $\quad$ N. Al-Azzawi, Baghdad University, IRQ

- NC. Basjaruddin, Politeknik Negeri Bandung, ID

- $\quad$ N. Che Pa, Universiti Putra Malaysia, MY

- N. Ifada, University of Trunojoyo Madura, ID

- $\quad$ N. Ojha, Sharda University, Greater Noida, UP, IND

- N. Prabaharan, SASTRA Deemed University, IND

- O. Ridha , University of Baghdad, IRQ

- O. Manad, UMANIS SA, FR

- P. Corral, Miguel Hernández University, ES

- P. Crippa, Università Politecnica delle Marche, ITA

- P. Mursanto, Universitas Indonesia, ID

- P. Zhang, Stony Brook University, USA

- R. Agustin, Universitas Pasundan, ID

- R. Fyath, Al-Nahrain University, College of Engineering, IRQ

- RC. Herrera Lara, National Polytechnic School, EC

- R. Kusumaningrum, Diponegoro University, ID

- R. Ngadiran, Universiti Malaysia Perlis, MY

- R. Nugroho, Soegijapranata Catholic University, AU

- R. Palma-Orozco, Instituto Politécnico Nacional, MX

- S. Alkassar, Ninevah University, IRQ

- S. Birla, Manipal University Jaipur, IND

- S. Biryuchinskiy, Vigitek, Inc, USA

- S. Horng , National Taiwan University of Science and Technology, TW 
- S. Mardiyanto, Institut Teknologi Bandung, ID

- $\quad$ S. Nazir, University of Peshawar, PAK

- S. Ooi, Multimedia University, MY

- S. Sarkar, Vijaya Vittala Institute of Technology, IND

- $\quad$ S. Singh, Manipal Institute of Technology, IND

- $\quad$ S. Suryono, Diponegoro University, ID

- $\quad$ S. Tadisetty, Kakatiya University College of Engineering and Technology, IND

- $\quad$ S. Tan, Newcastle University, GB

- T. Chen, MAXEDA Technology, TW

- T. Manjunath, Dayananda Sagar College of Engineering, Bangalore, Karnataka, IND

- T. Nguyen, Purdue University Fort Wayne, USA

- T. Yingthawornsuk, King Mongkut's University of Technology Thonburi, THA

- V. Ramos, Universidad Autonoma Metropolitana, MX

- V. Omwenga, Strathmore University, KEN

- W. Sunindyo, Institut Teknologi Bandung, ID

- W. Suwarningsih, Indonesian Institute of Science, ID

- Y.Su, Shu-Te University, TW

- Z. Mekkioui, University of tlemcen, DZ 


\section{Uncertainty Avoidance and Individualism Collectivism on Acceptance of Smart City Mobile Applications}

\author{
Julius Galih Prima Negara* \\ a Sistem Informasi \\ ${ }^{b}$ Magister Teknik Informatika \\ Universitas Atma Jaya Yogyakarta \\ Yogyakarta, Indonesia \\ *Corresponding author: \\ julius.galih@uajy.ac.id
}

\author{
Djoko Budiyanto Setyohadi \\ Magister Teknik Informatika \\ Universitas Atma Jaya Yogyakarta \\ Yogyakarta, Indonesia \\ djoko.bdy@gmail.com
}

\author{
Pranowo \\ Magister Teknik Informatika \\ Universitas Atma Jaya Yogyakarta \\ Yogyakarta, Indonesia \\ pranowo@uajy.ac.id
}

\begin{abstract}
The issue of e-government development and the concept of the smart city as an answer the government service challenges have emerged before. Cellular communication technology, communication networks, are developing rapidly and provide opportunities to play a role in the development of government areas. The emergence of smart city mobile applications can play a role in solving the challenges of shortening time, cutting distances, and disclosure of information. A technology acceptance survey has been carried out combined with cultural moderators. The survey data in Yogyakarta, Indonesia, is processed using Smart PLS 3.2 with Sequential Equation Modeling. It is unique to be discussed because the p-values of Uncertainty Avoidance (UC) 0,013 which means this is most significant, but Individual/Collectivism (IC) is more than 0.05 , which means IC is not significant, it does not moderate the behavior of users of this technology. In this paper, we describe the findings of IC research that do not moderate behavioral intentions. One of our findings is that users use this application due to government service needs, so aspects of individualism and collectivism have no significant effect. We review other findings in the IC moderator in-depth about why they do not affect.
\end{abstract}

Keywords - individualism, collectivism, smart city, mobile applications, acceptance

\section{INTRODUCTION}

A city today is a complex system characterized by a large number of interconnected citizens, businesses, various modes of transportation, communication networks, services, and utilities. In their appearance, smart cities are the answer to this challenge. Connecting many people, meeting intelligent transportation needs, facilitating communication, improving government performance, and making some aspects of the life of "smart" people embody a smart city. A smart city is also presented to address coordination issues between various levels of administration, as well as between government and society [1]. Developing smart cities with the latest technology can solve various challenges and problems in Indonesia. Investigating a government mobile product or application becomes very interesting to find out the value of the technology's acceptance.

Smart City is currently seen as a critical strategy to improve the quality of life of a large population of people living in cities throughout the world [2]. This system and service initiative can provide meaningful improvements to increase the productivity of governments, the private sector, and citizens. While traditional users of government services have had a passive role in the past, today's users are more active, more informed, and more demanding. We consider that e-government research, especially smart cities, is very necessary for developing countries as stated by [3].

The author has researched the acceptance of smart city mobile applications combined with cultural moderators. In this study, the discovery of several significant variables from the UTAUT2 Model, and the Hofstede cultural moderator construct moderate moderating behavioral intentions. There are interesting findings that we reveal are not the same as previous studies.

In this finding, individualism and collectivism do not moderate one's behavioral intentions in accepting and using technology. In countries with high collectivism scores, it is easier for people to adopt new technologies [4], [5]. This scientific paper will investigate why this cultural construct does not affect.

\section{THEORETICAL BACKGROUND}

\section{A. Smart City}

In the 1990s, the concept of Smart City first appeared [6]. At that time, Smart City was featured as an alternative to the traditional model, using new technology (especially ICTs) to overcome various challenges. Urban challenges that are increasingly urban are believed to be faced with this new concept. There are several perspectives on this concept, one of these is the perspective on the development of the city must consider the linkages between infrastructure, society, and institutions. Authors who applied this holistic Smart City concept in their research proposals [6]-[8]. Because this concept connects the residents in the city, we assume it interesting, why IC does not moderate behavioral intentions.

\section{B. Acceptance Model}

Among the many models proposed have been dominated by five theoretical models [9], until 2003, Venkatesh in [10] introduced the UTAUT theory. The five theories include IDT, TRA, TPB, TAM, and TPR. Innovation Diffusion Theory or abbreviated as IDT is explained in research [11]. The Theory of Reasoned Action was known as TRA in 1975. TPB, which stands for the Theory of Planned Behavior [12]. The Technology Acceptance Model that is often used today is called (TAM) [13]. The last of the five theories is the Perception Risk Theory (TPR) [13].

The unified theory of acceptance and use of technology in 2003 emerged from Venkatesh's ideas [10], building a substantial new basic model for technology acceptance studies. Below this will explain the Unified Theory of Acceptance and Use of Technology. 
UTAUT proposes four constructs [10], first is performance expectation, second is effort expectation, third is social influence, and the for the last facilitation conditions. Since this model existed, this model was gradually attracted by researchers. It was recently implemented to explore user acceptance of cellular technology, and gradually test the acceptance of several technologies in the use of individuals and organizations in several countries [14].

A good and detailed model has been offered for the exploration of the acceptance and use of technology by UTAUT, but exactly limitations following it [15]. UTAUT2 in 2012 was developed as an answer to expand and adapt the theory of consumer contexts [16]. Seven constructions have been owned, as indicated in the Model (Fig. 1). These are: performance expectations, effort expectation, social influence, facilitation conditions, hedonic motivation, price value, and seventh is habit. Added three constructs that did not exist before; the first predictor is hedonic motivation. Usually, the user will bear the costs of the service he has used, so construction is added to the price value. Then habits are also included as predictors.

Gender, age, and experience are moderate variables that influence and eliminate volunteerism from the previous UTAUT. Facilitation conditions and habits are also hypothesized to add a direct relationship with user behavior and behavioral intentions. User experience moderates the effects of behavioral intentions on usage, in addition to those we mentioned earlier above.

\section{Cultural Moderators}

It is believed that culture influences the way people use information systems [14], and some researchers write in the technology acceptance model this factor needs to be included [17] because cultural values influence being a moderator in acceptance [18]. We conducted several searches for scientific articles on this theme. We are seeing a continuous increase in academic research with regard to technology and cellular use culture. However, we see a few that combine the two things. To define culture we can use several ways, and several points of view. One point of view for example is about how people solve problems and carry out reconciliation in society.

We can open our eyes and accept new opinions that the importance of research that combines technology acceptance theory and cultural moderation is interesting [19]. In his classification, he explained individualism/collectivism, which means people can take care of themselves or their close relatives and can sort out which is more important than personal interests or social interests. The second classification is power distance, which means how the reality of the distribution of power is not evenly shared by the community. Next, there is the avoidance of uncertainty as to the extent to which the community feels uncomfortable or threatened by uncertain and ambiguous situations, then the community or individual avoids it.
Data from more than 50 countries were collected by Hofstede and IBM, and published cultural dimensions. Indonesia, as a developing country, has the following values: 48 uncertainty avoidance, 14 individualism/collectivism, 46 masculinity/femininity, 68 long-term orientation, and 78 in power distance [20]. It means that the area is very hierarchical, collectivist, low masculine, low uncertainty avoidance, and long-term oriented.

Some people disagree that the cultural dimension is used for purposes other than country-level studies [4] or which means it is inappropriate to use cultural dimension scores in countries to predict individual behavior. Nevertheless, it has been adopted the cultural dimension used at the individual level [21].

\section{RESEARCH MODEL AND HYPOTHESIS}

UTAUT 2nd generation or as we know it as UTAUT2, and Hofstede's cultural moderators are combined in this research as a theoretical basis. Previous research revealed that compared to the previous model UTAUT2 showed a substantial increase in variance that could explain usage intentions and behavior [16] therefore we consider it compatible with the aims of this study. Because we see that society has been using information systems because it is influenced by habits and culture, we use Hofstede's moderator in the research and we report the results in this paper. Performance expectations or what we call PE in this study are the level of confidence of a user that the smart city mobile application will have a positive impact or benefit for him in the context of government services [10]. In one of these studies we also found that people will use technology if they believe they will get positive things [22].

Acceptance of risk is often taken with relaxed and no doubt by people in countries with low uncertainty avoidance [20], and the adoption of IT technology or something new will be tried as a result [23]. Because of this we have a hypothesis:

H1. Behavioral intention (BI) and usage behavior (UB) are moderated by Uncertainty avoidance (UC), people with higher uncertainty avoidance, the weaker the level of moderation.

Collectivist groups have more attachment to one another and maintain cohesiveness to the group. People in collectivist groups will listen more to the opinions of others about the use of technology. Unlike the above, a country or region that has an individualist culture has a higher level of egoism than a society that has a collectivism culture [24]. New services or new technology will be easily accepted by the public in a collectivist country like Indonesia [4]. Because of this we have a hypothesis : 


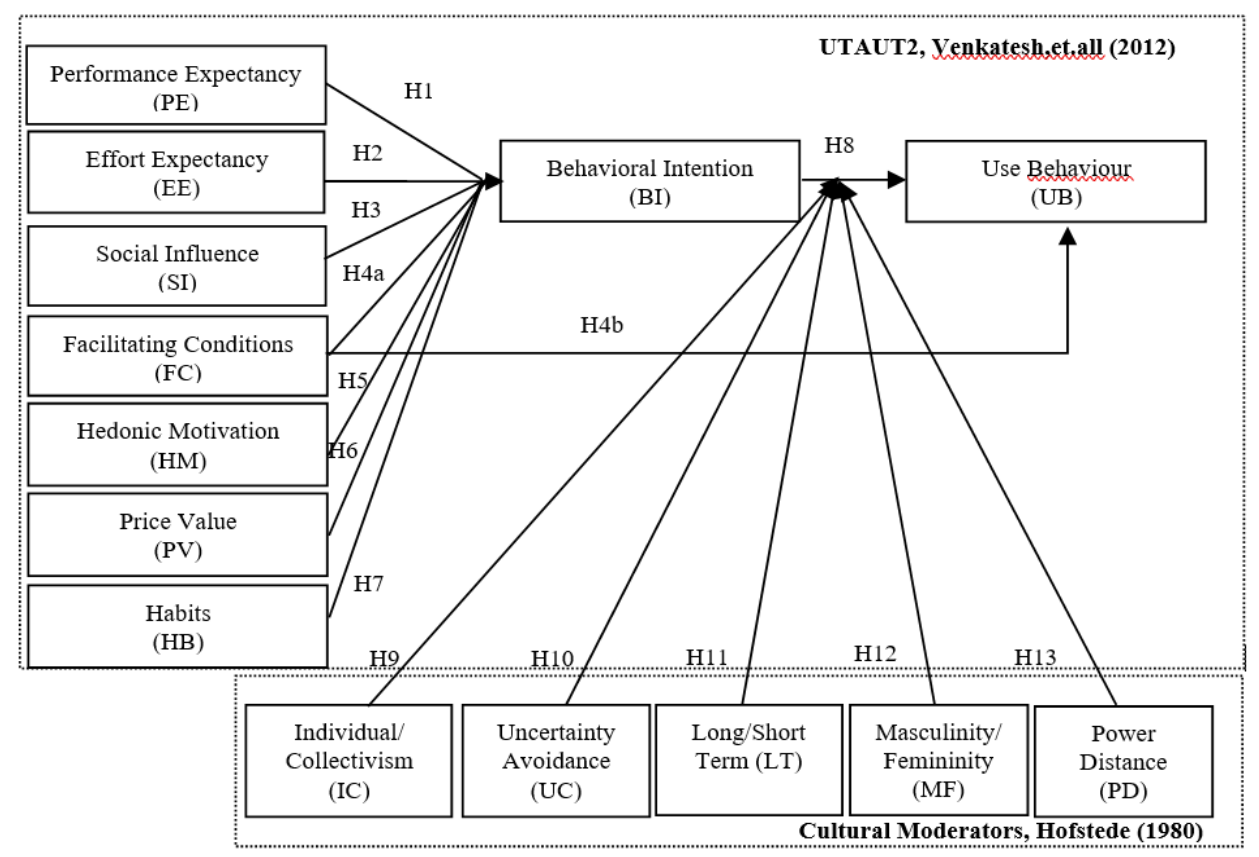

Fig. 1. Research Model

H2. Behavioral intention (BI) and usage behavior (UB) are moderated by Individualism/collectivism (IC) so that people in areas that have a collectivist culture will have a stronger relationship. In Figure Fig.1, this research model is explained. The model used by this previous study is a combination of the UTAUT2 acceptance model and the cultural moderator Hofstede

\section{Data Collections AND Research Methodologies}

Data collection was carried out in Yogyakarta, Indonesia. In the context of consumers, the use of Jogja Smart Service services is an entirely voluntary decision, not because of work obligations or other compelling reasons. The questionnaire is in English and has been reviewed for validity by a group of academics.

In this study, there are different parts: the first is the construction of technology acceptance data using the UTAUT2 model, the second is culture as a parameter, the third is for general information. The items and scale for UTAUT2 construction were adapted from [10], [16], use behavior from [25], and individualism / collectivism from [18]. Each item is measured on a five-point Likert scale, ranging from 'strongly disagree'(1) to ' 'strongly agree' (5). Martins write usage behavior is coded from 1 (never) to 11 (several times per day) [25], in accordance with the effective use of Jogja Smart Service. The questionnaire was translated into Indonesian, sent to academics graduates of the English Language Study Program to be reviewed and corrected at any time according to the characteristics of the local Indonesian language. With different people we translated the questionnaire back into English to ensure consistency and validation [26].

Based on the fact that studies on technology acceptance have traditionally been carried out using survey research [10], a survey was conducted by distributing questionnaires to the research target. Thirty individual Jogja Smart Service users were used to test this survey. This is not included in the final data. Reliable and validity are shown by the scale as preliminary evidence. The snowball sampling pattern is used in distributing questionnaires to make it easier to get respondents using this application. We do this because not all people of Yogyakarta City have used this application until the research was conducted. So we decided on this method to get recommendations on who uses Jogja Smart Service.

TABLE I. DESCRIPTIVE STATISTICS OF RESPONDENTS

\begin{tabular}{|c|c|c|c|}
\hline Measure & Value & Frequency & $\%$ \\
\hline \multirow[t]{2}{*}{ Gender } & Male & 180 & 64 \\
\hline & Female & 101 & 36 \\
\hline \multirow[t]{3}{*}{ Age } & Bellow 35 & 168 & 60 \\
\hline & $36-50$ & 88 & 31 \\
\hline & Over 50 & 25 & 9 \\
\hline \multirow[t]{3}{*}{ Education } & Lower than bachelor & 180 & 64 \\
\hline & Bachelor & 96 & 44 \\
\hline & Master or higher & 5 & 2 \\
\hline \multirow{6}{*}{$\begin{array}{l}\text { Smartphone Use } \\
\text { frequency }\end{array}$} & Bellow & 0 & 0 \\
\hline & between 1-3 & & \\
\hline & hours a day & 43 & 15 \\
\hline & $\begin{array}{l}\text { between } 3-5 \\
\text { hours a day }\end{array}$ & 107 & 38 \\
\hline & $\begin{array}{l}\text { between } 5-7 \\
\text { hours a day }\end{array}$ & 121 & 43 \\
\hline & $\begin{array}{l}\text { over } 7 \text { hours } \\
\text { a day }\end{array}$ & 10 & 4 \\
\hline \multirow{7}{*}{$\begin{array}{l}\text { Internet Use } \\
\text { Frequency }\end{array}$} & Bellow & & \\
\hline & 1 hour a day & 0 & 0 \\
\hline & $\begin{array}{l}\text { between 1-3 } \\
\text { hours a day }\end{array}$ & 14 & 5 \\
\hline & between $3-5$ & & \\
\hline & hours a day & 96 & 34 \\
\hline & $\begin{array}{l}\text { between } 5-7 \\
\text { hours a day }\end{array}$ & 147 & 52 \\
\hline & $\begin{array}{l}\text { over } 7 \text { hours } \\
\text { a day }\end{array}$ & 24 & 9 \\
\hline
\end{tabular}


At the end of October 2019, a total of 281 valid answers were collected after 12 weeks. The Kolmogorov-Smirnov (KS) test was carried out to see the sampledistribution of the first and second groups of respondents. Then verify and ensure that the two are not statistically different. It indicates not existing non-response bias. Table 1 presents the respondent's data and is described in detail with descriptive statistics.

\section{RESUlt AND DATA ANALYSIS}

The depiction of the statistical model used to evaluate the validity of substantive theories with empirical data is SEM, which stands for Structural Equation modeling, which has been widely used [27]. Covariance-based and variant-based techniques are two of the techniques that exist today. We performed theoretical tests using variant-based techniques. We did this test using Partial Least Square (PLS) software called SmartPLS 3.0. This is an excellent statistical technique and is considered suitable for many research situations [28], for many constructs in a complex model is suitable.

We aim to construct a sample dimension that is ten times larger than the maximum of the path [29], and therefore PLS can be considered adequate for estimation. When we compared with other SEMs such as covariance-based techniques, this technique is known to have minimal restrictions in terms of sample size and residual distribution. The guidelines were followed, and the analysis was carried out in two steps [30]; we begin with an assessment of the reliability and validity of the measurement model, followed by an assessment of the structural model and testing the hypothesis. These two steps are described in the steps below.

\section{A. Model Measurement}

Discriminant validity, convergence validity, reliability indicators, and construct reliability are included in the measurement model. In Table 2 provides information on AVE, composite reliability abbreviated as $\mathrm{CR}$, Cronbach Alpha abbreviated as CA, loadings, and t-value. All constructions have reliability and composites, as shown in the table, CA was indicating construction reliability because of the value greater than 0.7 [31]. The reliability of the indicator is evaluated based on the criteria that loadings must be higher than 0.7 and that any loadings below 0.4 must be removed [32]. Due to the low loadings factor, some items are dropped. 0.7 or higher is the remaining load and was statistically significant at 0.05 . AVE is used to test the convergence validity, and then all constructs are positively compared with the minimum value that can be received from 0.50 [33] and [34], as seen in Table 2.

The cross-loading factor and Fornell-Larcker criteria are used to analyze discriminant validity. Instead of each crossloading on other factors, each item presents a higher loading on the corresponding factor. Some items must be removed from each variable to ensure cross-loading criteria. Evidence of the validity of the discriminant scale is proven because two criteria are ultimately fulfilled. It was confirmed that constructs were statistically different and could be used to test structural models. This is because of discriminant validity, convergence validity, indicator reliability, good construct reliability are shown by the results of the model measurements.
TABLE II. FACTOR LOADINGS AND QUALITY CRITERIA

\begin{tabular}{cccccccc}
\hline Construct & AVE & CR & CA & & \multicolumn{2}{c}{ Item Loadings } & t-value \\
\hline \multirow{2}{*}{ BI } & 0,899 & 0,947 & 0,887 & BI2 & 0,952 & 52,669 \\
& & & & BI3 & 0,944 & 71,726 \\
& & & & IC1 & 0,896 & 16,749 \\
IC & 0,708 & 0,878 & 0,791 & IC2 & 0,866 & 12,931 \\
& & & & IC4 & 0,755 & 10,836 \\
& & & & & UC2 & 0,829 & 19,819 \\
\multirow{2}{*}{ UC } & \multirow{2}{*}{0,778} & 0,778 & 0,857 & UC3 & 0,916 & 23,087 \\
& & & & UC4 & 0,898 & 21,219 \\
\hline
\end{tabular}

TABLE III. PATH COEFFICIENTS AND THEIR SIGNIFICANCE

\begin{tabular}{cccccc}
\hline & Hypothesis & $\begin{array}{c}\text { Original } \\
\text { Sample } \\
(\mathbf{O})\end{array}$ & $\begin{array}{c}\text { Sample } \\
\text { Mean } \\
(\mathbf{M})\end{array}$ & $\begin{array}{c}\text { T } \\
\text { Statistics } \\
(\mid \mathbf{O} / \mathbf{S T D E} \\
\mathbf{V} \mid)\end{array}$ & $\begin{array}{c}\text { p- } \\
\text { values }\end{array}$ \\
\hline $\begin{array}{c}\text { Moderator } \\
\text { IC }\end{array}$ & $\mathrm{H} 2$ & 0,021 & 0,021 & 0,586 & $\mathbf{0 , 5 5 8}$ \\
$\begin{array}{c}\text { Moderator } \\
\text { UC }\end{array}$ & $\mathrm{H} 1$ & 0,111 & 0,107 & 2,602 & $\mathbf{0 , 0 1 3}$ \\
\hline
\end{tabular}

\section{B. Hypotheses Testing and Structural Model}

The examination of standard pathways is based on hypothesis analysis and relationship building. Bootstrap resampling is used to estimate the level of significance of the path [28], with 500 iterations of resampling. The results are summarized in Table 3.

This model explains $57.2 \%$ of the variation in behavioral intentions and $72.4 \%$ in user behavior. Behavioral intentions and use behavior moderated by uncertainty avoidance and statistically appear with $p$ value $<0.01$. Surprisingly, individualism and collectivism have the highest $\mathrm{p}$-values from the standards of moderation.

\section{DISCUSSIONS}

Incorporating the theory of acceptance of UTAUT2, from [16], with a cultural moderator from [4], is a unique model way, to explain the acceptance of Jogja Smart Service. This research captures the cultural phenomenon in moderating the use of technology.

\section{A. Main Findings}

There are interesting things found when the results of this study have appeared. In some cultural moderator variables, only one construct is UC, which moderates behavioral intentions. Surprisingly Individualism and Collectivism do not influence or moderate behavioral intentions. The P-values of IC's raised in the results of this study were 0.558 . This score means the p-values did not support the moderation of behavioral intentions for user behavior. This finding contradicts previous research, which said that IC moderated significantly. Our initial suspicion is that research was carried out when the release of this application was only carried out for one year and is still in the process of migrating from conventional services to integrated services.

A society with low individualism or high collectivism value should be easy to recommend someone to use technology that has been used. Nevertheless, this application 
was just released and developed gradually. In the findings of this study, it is assumed that the moderator of UC (Uncertainty Avoidance) moderates behavioral intentions in usage behavior. This happens because the purpose of the smart city mobile application is to cut the distance between the government and the community, shorten service times, and answers to the challenges of information disclosure. This construct is appropriate because people with low levels of uncertainty avoidance will be easier to accept and use new technology.

The most important finding from this study is that IC is no more significant than UC. Further research can be done again after this application is not in the stage of introduction to the community. Because it can be explored further whether people using this mobile application are influenced by factors of Indonesia as a society with a high collectivism score. With this collectivism, it is believed that the community will be influenced by those around them. The government's goal in providing information certainty is achieved. It is also proven that the public uses technology because it seeks information certainty.

\section{B. Supported findings}

The uncertainty avoidance construct is the only one that has a positive value, and moderates behavioral intentions towards JSS usage behavior. As in [20], it was stated that in countries with low uncertainty avoidance, people are not afraid to take risks and are relaxed, and consequently, they are easier to try something and also try new IT technologies that are offered [23]. This is in line with the phenomenon in Indonesia that several technology companies have made Indonesia a significant target market.

The citizens will adopt the technology he has to the specifications needed by the software he will install. Instead of returning to conventional methods of government service, they take the opportunity to get the convenience of installing the latest mobile applications. One of them applies to egovernment or smart city technology published by the government. Therefore if the government wants the latest technology adopted, it must pay attention to aspects of facilitating conditions [35].

People in Indonesia will quickly switch from current services to the latest government services if the negatives of old services are solved by technology. People who were reluctant to come to the government office in the past will be happy with this software because this service is ubiquitous. The community feels happy and happy because government services are no longer boring [36].

This study, with its model, validates the influence of the Hofstede cultural moderator on behavioral intention and usage behavior. Individualism/collectivism has a weak and did not have a moderate effect in line with some studies[18], but there is one research in the opposite [4].

\section{Additional Findings}

During the research, the Yogyakarta's government was still in the stage of developing and introducing this mobile application. It is possible that in the time of migration from conventional services to smart city services, the citizen did not know deeply. Only a few are installed to meet their needs and only a smaller number suggests someone uses this application. As time went by, after several people felt the benefits of a new government application, they were trusted to provide suggestions to other people to use JSS. Given the collectivist characteristics of Indonesian society, the government should see this as an opportunity to challenge technology recommendations to others.

In our study, we did not fully confirm the constructions contained in UTAUT2 that were important. Effort expectancy does not influence the use of technology. Users feel the smart city application is easy to get and use, so users assume they will not get a problem because they are already proficient with technology. Social influence was found to be inconsistent with previous academic publications [37], the results were supposed to support cultural influences, namely collectivism and the distance of power. However, in this research in Indonesia, it was found that collectivism did not moderate the behavioral intention to use behavior. In Indonesia, as a collectivist society, it is hoped that social influence can play a significant role in this intention because individuals who are close to the community will accept opinions and even ask for input from others [38]

\section{CONCLUSION}

This study shows that Individualism and collectivism are not significant variables. This happens because the smart city mobile application, at the time of the research, it was still in the process of migrating from current government services to mobile applications. This causes not all people to know and use this application and just a few who recommend each other. The citizens have not yet made recommendations for others in using this government mobile application. UC still holds the highest score in moderating usage, i.e., the low avoidance community will no doubt try, new technology. This is like confirming several studies that the impact of personal users can be influenced by end-user computing satisfaction like this study [39].

Furthermore, the UC variable shows that people are motivated to use because they are looking for certainty in government services. The results of research like this can be used to reduce knowledge gaps to prevent further failures [40]. For further exploration, research can be done about the influence of IC moderation on government applications that have long been used or have been widely used by the community. In the end, the contribution to the government from this research is that the government knows that the UC aspect is vital because people will readily accept new technology and will not be afraid to try it when it suits their needs. The IC aspect can be a second priority approach in promoting a government mobile application.

\section{REFERENCES}

[1] M. Angelidou, "Smart city policies: A spatial approach," Cities, vol. 41, pp. S3-S11, 2014, doi: 10.1016/j.cities.2014.06.007.

[2] H. Ahvenniemi, A. Huovila, I. Pinto-Seppä, and M. Airaksinen, "What are the differences between sustainable and smart cities?," Cities, vol. 60, pp. 234-245, 2017, doi: 10.1016/j.cities.2016.09.009.

[3] J. D. Twizeyimana and A. Andersson, "The public value of EGovernment - A literature review," Government Information Quarterly, vol. 36, no. 2. pp. 167-178, 2019, doi: 10.1016/j.giq.2019.01.001. 
[4] G. Hofstede, Culture's consequences: International differences in work-related values. Beverly Hills: Sage Publications, 1980.

[5] G. Baptista and T. Oliveira, "Understanding mobile banking: The unified theory of acceptance and use of technology combined with cultural moderators," Comput. Human Behav., vol. 50, pp. 418-430, 2015, doi: 10.1016/j.chb.2015.04.024.

[6] S. Alawadhi et al., "Building understanding of smart city initiatives," Lect. Notes Comput. Sci. (including Subser. Lect. Notes Artif. Intell. Lect. Notes Bioinformatics), vol. 7443 LNCS, pp. 40-53, 2012, doi: 10.1007/978-3-642-33489-4_4.

[7] A. Caragliu, C. del Bo, and P. Nijkamp, "Smart cities in Europe," $J$. Urban Technol., vol. 18, no. 2, pp. 65-82, 2011, doi: 10.1080/10630732.2011.601117.

[8] V. Fernandez-Anez, J. M. Fernández-Güell, and R. Giffinger, "Smart City implementation and discourses: An integrated conceptual model. The case of Vienna," Cities, vol. 78, no. June, pp. 4-16, 2018, doi: 10.1016/j.cities.2017.12.004

[9] H. Hoehle, E. Scornavacca, and S. Huff, "Three decades of research on consumer adoption and utilization of electronic banking channels: A literature analysis," Decis. Support Syst., vol. 54, no. 1, pp. 122 132, 2012, doi: 10.1016/j.dss.2012.04.010.

[10] F. D. Venkatesh, Viswanath. Morris, Michael G. Davis, Gordon B. Davis, "User Acceptance of Information Technology: Toward a Unified View," Microvasc. Res., vol. 27, no. 3, pp. 425-478, 2003, doi: 10.1006/mvre.1994.1019.

[11] E. M. Rogers, "Diffusion of Innovations: Modifications of a Model for Telecommunications," Die Diffus. von Innov. der Telekommunikation, pp. 25-38, 1995, doi: 10.1007/978-3-642-798689 2.

[12] I. Ajzen, "The theory of planned behavior," Handb. Theor. Soc. Psychol. Vol. 1, pp. 438-459, 1991, doi: 10.4135/9781446249215.n22.

[13] F. D. Davis, "Information Technology Introduction," vol. 13, no. 3, pp. 319-340, 1989.

[14] I. Im, S. Hong, and M. S. Kang, "An international comparison of technology adoption: Testing the UTAUT model," Inf. Manag., vol. 48, no. 1, pp. 1-8, 2011, doi: 10.1016/j.im.2010.09.001

[15] A. Negahban and C. H. Chung, "Discovering determinants of users perception of mobile device functionality fit," Comput. Human Behav., vol. 35, pp. 75-84, 2014, doi: 10.1016/j.chb.2014.02.020.

[16] V. Venkatesh, J. Y. . Thong, and X. Xu, "Consumer Acceptance and Use of Information Technology: Extending the Unified Theory of Acceptance and Use of Technology," MIS Q., vol. 36, no. 1, p. 157, 2012, doi: 10.2307/41410412.

[17] J. Park, "Adoption of Mobile Technologies for Chinese Consumers," J. Electron. Commer. Res., vol. 8, no. 3, p. 196, 2007.

[18] M. Srite and E. Karahanna, "The Role of Espoused in Technology Values National Cultural," Mis Q., vol. 30, no. 3, pp. 679-704, 2006.

[19] F. Trompenaars and H. C. -Turner, Riding the Waves of Culture Understanding Cultural Diversity in Business. 1993.

[20] G. Hofstede, "The Hoftsede Insight," 2019. https://www.hofstedeinsights.com/country/indonesia/.

[21] B. L. Kirkman, K. B. Lowe, and C. B. Gibson, "A quarter century of culture's consequences: A review of empirical research incorporating Hofstede's cultural values framework," J. Int. Bus. Stud., vol. 37, no. 3, pp. 285-320, 2006, doi: 10.1057/palgrave.jibs.8400202.

[22] C. A. Compeneau, D. R.Higgins, "Development of a measure and initial test," Manag. Inf. Syst., vol. 19, no. 2, pp. 189-211, 2012.
[23] I. P. L. Png, B. C. Y. Tan, and K. L. Wee, "Dimensions of national culture and corporate adoption of IT infrastructure," IEEE Trans. Eng. Manag., vol. 48, no. 1, pp. 36-45, 2001, doi: 10.1109/17.913164.

[24] A. Ben Zakour, "Cultural Differences and Information Technology Acceptance," South. Assoc. Inf. Syst., pp. 156-161, 2004.

[25] C. Martins, T. Oliveira, and A. Popovič, "Understanding the internet banking adoption: A unified theory of acceptance and use of technology and perceived risk application," Int. J. Inf. Manage., vol. 34, no. 1, pp. 1-13, 2014, doi: 10.1016/j.ijinfomgt.2013.06.002.

[26] R. W. Brislin, Back translations for cultural research. 1970.

[27] C. M. Ringle, S. Wende, and A. Will, "Smart PLS 3.0." 2019, [Online]. Available: https://www.smartpls.com/downloads.

[28] J. Henseler, C. M. Ringle, and R. R. Sinkovics, "The use of partial least squares path modeling in international marketing," $A d v$. Int. Mark, vol. 20, no. 2009, pp. 277-319, 2009, doi: 10.1108/S14747979(2009)0000020014.

[29] D. Gefen and D. Straub, "A Practical Guide To Factorial Validity Using PLS-Graph: Tutorial And Annotated Example," Commun. Assoc. Inf. Syst., vol. 16, no. July, 2005, doi: 10.17705/1 cais.01605.

[30] J. C. Anderson and D. W. Gerbing, "Structural Equation Modeling in Practice: A Review and Recommended Two-Step Approach," Psychol. Bull., vol. 103, no. 3, pp. 411-423, 1988, doi: 10.1037/00332909.103.3.411.

[31] D. Straub, "Validating Instruments in MIS Research," MIS Quart., vol. 13, pp. 147-169, 1989.

[32] G. A. Churchill, "A Paradigm for Developing Better Measures of Marketing Constructs," J. Mark. Res., vol. 16, no. 1, p. 64, 1979, doi: $10.2307 / 3150876$.

[33] C. Fornell and D. F. Larcker, "Evaluating Structural Equation Models with Unobservable Variables and Measurement Error," J. Mark. Res., vol. 18 , no. 1, p. 39, 1981, doi: 10.2307/3151312

[34] J. Henseler, C. M. Ringle, and R. R. Sinkovics, "The use of partial least squares path modeling in international marketing," $A d v$. Int. Mark, vol. 20, no. 2009, pp. 277-319, 2009, doi: 10.1108/S14747979(2009)0000020014.

[35] D. Gilbert et al., "Transforming Government: People , Process and Policy Article information :," 2009.

[36] L. Zhang, J. Zhu, and Q. Liu, "A meta-analysis of mobile commerce adoption and the moderating effect of culture," Comput. Human Behav. vol. 28, no. 5, pp. 1902-1911, 2012, doi: 10.1016/j.chb.2012.05.008.

[37] L. Wang and Y. Yi, "The impact of use context on mobile payment acceptance: An empirical study in China," Adv. Intell. Soft Comput., vol. 140 AISC, pp. 293-299, 2012, doi: 10.1007/978-3-642-279454 47.

[38] L. Putit and D. Arnott, "Micro-culture and consumers adoption of technology: A need to re-evaluate the concept of national culture," Acad. Mark. Sci. Rev., vol. 11, no. 6, 2007.

[39] T. L. M. Suryanto, D. B. Setyohadi, and N. C. Wibowo, "Empirical investigation on factors related to individual of impact performance information system," Int. Conf. Electr. Eng. Comput. Sci. Informatics, vol. 2017-Decem, no. September, 2017, doi: 10.1109/EECSI.2017.8239123.

[40] T. Choi and S. M. Chandler, "Knowledge vacuum: An organizational learning dynamic of how e-government innovations fail," Government Information Quarterly, vol. 37, no. 1. 2020, doi: 10.1016/j.giq.2019.101416. 